\title{
Frontières
}

\section{La douleur chez les personnes ayant des déficits cognitifs}

\section{Un double isolement}

\section{Thérèse Audet et Marie-Josée Turgeon}

Volume 13, numéro 2, printemps 2001

Les morts de l'esprit

URI : https://id.erudit.org/iderudit/1074457ar

DOI : https://doi.org/10.7202/1074457ar

Aller au sommaire du numéro

\section{Éditeur(s)}

Université du Québec à Montréal

ISSN

1180-3479 (imprimé)

1916-0976 (numérique)

Découvrir la revue

Citer cet article

Audet, T. \& Turgeon, M.-J. (2001). La douleur chez les personnes ayant des déficits cognitifs : un double isolement. Frontières, 13(2), 50-53.

https://doi.org/10.7202/1074457ar
Résumé de l'article

La douleur ressentie par une personne est une expérience subjective. Le personnel soignant qui s'occupe des personnes souffrantes doit mettre en branle un processus d'inférence puisqu'il existe peu de moyens fiables pour évaluer l'intensité réelle de la douleur. Une douleur mal exprimée engendre une mauvaise évaluation de cette douleur et risque alors d'être mal soulagée. Cette situation est fort susceptible de se produire dans les cas de démence ou dans les cas de déficience intellectuelle, puisque ces personnes ne peuvent exprimer clairement leur douleur. Il faut alors recourir à des outils particuliers pour l'évaluer, sinon l'isolement, que leur confèrent déjà leurs déficits cognitifs, s'accentue, car les comportements difficiles qu'engendre la douleur éloignent d'elles leur entourage. 


$\begin{array}{llllllllllll}\text { I } & N & T & E & R & V & E & N & T & \text { I } & O & N\end{array}$

\section{Résumé}

La douleur ressentie par une personne est une expérience subjective. Le personnel soignant qui s'occupe des personnes souffrantes doit mettre en branle un processus d'inférence puisqu'il existe peu de moyens fiables pour évaluer l'intensité réelle de la douleur. Une douleur mal exprimée engendre une mauvaise évaluation de cette douleur et risque alors d'être mal soulagée. Cette situation est fort susceptible de se produire dans les cas de démence ou dans les cas de déficience intellectuelle, puisque ces personnes ne peuvent exprimer clairement leur douleur. Il faut alors recourir à des outils particuliers pour l'évaluer, sinon l'isolement, que leur confèrent déjà leurs déficits cognitifs, s'accentue, car les comportements difficiles qu'engendre la douleur éloignent d'elles leur entourage.

Mots clés: douleur-déficits cognitifs

\section{Abstract}

Experiencing pain is a highly subjective feeling. Individuals who attend to persons in pain must often rely on inferences about that pain, since few measures are available to evaluate its real intensity. If pain is not clearly described, it will be incorrectly assessed and, consequently, poorly relieved. This problem is especially likely to arrive in patients with dementia or in patients with mental retardation who have difficulties in expressing clearly their feelings of pain. Special measures should be used to assess pain in these patients, or else their sense of isolation, already present because of their cognitive deficits, might grow deeper since behaviors associated with pain put a barrier between them and their surroundings.

Key words: pain-cognitive deficits

\section{La douleur chez les personnes ayant des déficits cognitifs Un double isolement}

\section{Thérèse Audet, Ph.D., Département de psychologie, Université de Sherbrooke.}

Marie-Josée Turgeon, M.Ps., Centre de réadaptation La Myriade.

La douleur est une réalité quotidienne pour beaucoup de personnes. C'est encore plus vrai pour les personnes âgées, car plusieurs maladies, dont l'incidence augmente avec l'âge, s'accompagnent de douleurs physiques chroniques. La qualité de vie des personnes qui souffrent en est affectée et l'un des moyens pour améliorer la situation passe par le soulagement de cette douleur.

Pour que le soulagement de la douleur soit efficace, il faut qu'une relation fructueuse s'établisse entre le personnel soignant et la personne souffrante. Or, les données sur le soulagement de la douleur, qui seront présentées ci-dessous font voir que cette relation est loin d'être idéale. Pour qu'elle le soit, il faudrait que la personne qui souffre exprime correctement sa douleur et que la personne qui l'écoute la perçoive telle qu'elle est.
Il n'est pas difficile d'imaginer, dans le cas particulier de personnes ayant des déficits cognitifs, comme c'est le cas dans la démence, ou comme c'est le cas dans la déficience intellectuelle, que la qualité de la relation entre le personnel soignant et la personne souffrante puisse être compromise. En effet, les difficultés que peuvent avoir ces personnes à exprimer leur douleur et la difficulté que peut avoir le personnel soignant à la percevoir constituent des obstacles à la relation. L'isolement qui peut en résulter devient un poids de plus pour la personne déjà privée de relations significatives à cause des déficits cognitifs qui entravent sa capacité à communiquer avec les autres. La douleur ressentie, mais non soulagée, vient accentuer la «mort de l'esprit » chez ces personnes, puisqu'elles ne peuvent même plus accéder à la quiétude ou au bien-être.

Quels sont donc les pièges qui peuvent tout particulièrement affecter la relation entre la personne souffrant de douleurs physiques et ayant des déficits cognitifs et le personnel soignant chargé de soulager cette douleur? La réponse à la question 
nécessite que soient d'abord rappelés les aspects subjectifs qui influencent l'expression et la perception de la douleur de manière générale. Ensuite, il faut présenter plus spécifiquement les difficultés particulières au traitement de la douleur des personnes ayant des atteintes cognitives.

\section{LES ASPECTS SUBJECTIFS \\ DE LA DOULEUR}

Chapman et Gavrin ${ }^{1}$ rapportent la définition de la douleur acceptée par l'Association internationale pour l'étude de la douleur, soit « une expérience sensorielle et émotionnelle désagréable, qui est associée à des lésions tissulaires, ces lésions étant réelles ou probables, ou qui est décrite en fonction de tels dommages ». Cette douleur peut être aiguë si elle est associée à une condition passagère ou chronique si elle est la résultante d'une condition physique plus permanente. Le simple fait que la définition parle d'une expérience émotionnelle laisse bien voir que le phénomène n'est pas que physique; la douleur peut envahir l'esprit au point de l'obnubiler. Cecily Saunders a justement développé le concept de douleur totale pour bien faire ressortir les aspects multidimensionnels de la douleur, soit les aspects physiques, psychologiques, sociaux et spirituels ${ }^{2}$.

Le soulagement de la douleur est un des principaux objectifs de la pratique

\section{LA DOULEUR PEUT ENVAHIR L'ESPRIT}

AU POINT DE L'OBNUBILER.

médicale et infirmière ${ }^{3}$. Pourtant, Barreau ${ }^{4}$ rapporte des chiffres étonnants; malgré le fait que les progrès relatifs aux modes de traitement permettent en principe de soulager entre 95 et $98 \%$ des douleurs, dans les faits, moins de $40 \%$ des douleurs sont soulagées. Comment un tel écart peut-il exister? La subjectivité qui entoure l'expression et la perception de la douleur est un des éléments importants qui sous-tend le problème du soulagement de la douleur.

Les moyens objectifs permettant d'évaluer avec exactitude l'intensité de la douleur, sans avoir à recourir à ce qu'en dit la personne qui souffre, n'existent pas encore, même si des signes comme l'accélération du pouls, la tension, le rythme de la respiration peuvent être des indicateurs de douleur. Davitz et Davitz ${ }^{5}$ soulignent que le personnel soignant doit inévitablement se tourner vers ce que la personne dit de sa douleur. Or, la douleur est une expérience unique et individuelle et elle n'est pas fonction seulement du degré réel de l'atteinte physiologique qui la sous-tend ${ }^{6}$. Les seuils de perception de la douleur, ainsi que la tolérance à la douleur, diffèrent d'une personne à l'autre et les antécédents culturels, de même que les expériences passées, influencent ces seuils ${ }^{7}$. Par exemple, chez certaines personnes âgées, les croyances religieuses à l'effet que supporter la douleur permet de gagner son ciel, peuvent contribuer à inhiber l'expression de la douleur. Leur tolérance à la douleur sera alors plus grande. D'autres, alors qu'elles étaient jeunes, ont été témoins de douleurs intolérables chez des gens de leur entourage, la peur de souffrir est tellement forte que la moindre douleur est exprimée et présentée comme étant excessive. Chez ces personnes, le seuil de tolérance à la douleur est bas.

Davitz et Davitz ${ }^{8}$ soulignent qu'en l'absence d'outils permettant de jauger exactement la douleur, la personne qui écoute la douleur exprimée par l'autre met en branle un processus d'inférence à l'égard de cette douleur. Or, ces inférences sont aussi empreintes de subjectivité, voire de préjugés. Par exemple, le simple fait que la personne qui souffre soit très jeune ou très âgée amène des croyances quant à la douleur. Visier ${ }^{9}$ rappelle la croyance qui

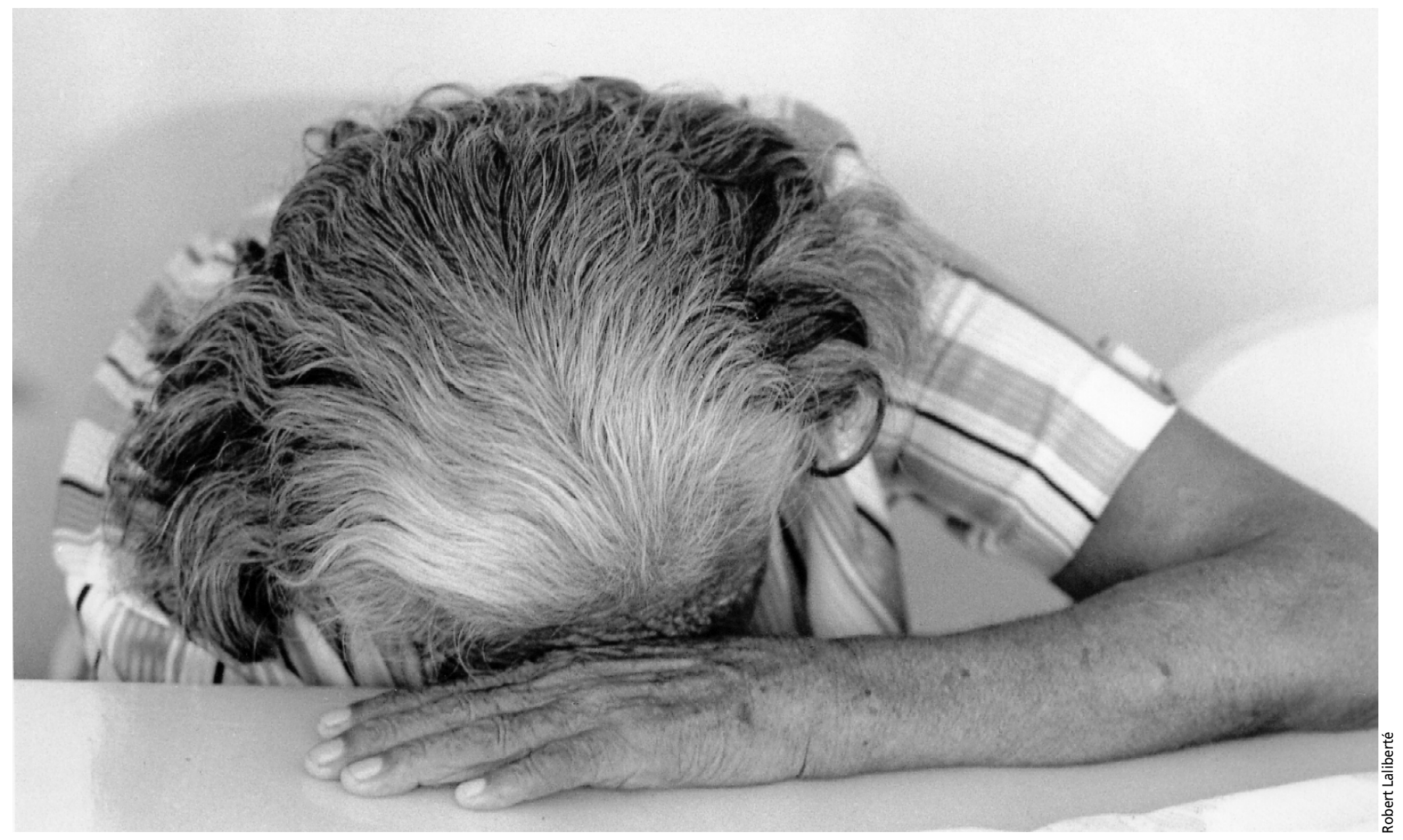


veut que les très jeunes enfants ne ressentent pas de douleur et Bouillé ${ }^{10}$ souligne l'existence du mythe qui dit que la sensation de douleur diminue avec l'âge (et donc que les personnes âgées souffrent moins). Des résultats de recherche montrent l'effet pervers de ces aspects subjectifs sur le soulagement de la douleur. Par exemple, pour les personnes âgées, Ross et Crook ${ }^{11}$ rapportent des données montrant que le personnel infirmier perçoit la douleur de grands brûlés âgés comme étant moins forte que celle de malades plus jeunes. Ces auteures rapportent aussi des données indiquant que les personnes âgées reçoivent moins d'analgésiques après une chirurgie ou encore pour le soulagement des douleurs cancéreuses que les patientes et les patients plus jeunes. Dans une recherche faite auprès de futurs médecins ${ }^{12}$, le fait de ne pas avoir de diagnostic connu pour expliquer une douleur entraîne une perception moins forte de cette douleur et une intention de la soulager qui est diminuée ${ }^{13}$.

La relation qui s'établit entre une personne qui souffre et le personnel soignant est fortement marquée par la subjectivité de la personne souffrante et par celle du personnel soignant. Il est donc primordial, pour que soit soulagée adéquatement la douleur, que la collaboration entre les partenaires puisse se faire, en respectant le champ de compétence de chacun. La personne qui souffre est celle qui peut décrire sa douleur et le personnel soignant qui l'écoute peut ensuite mettre en branle le soulagement de cette douleur. Toutefois, comme le soulignent Cook et Roy ${ }^{14}$, lorsque le système de croyances d'un des partenaires (la personne qui souffre) s'oppose au système de croyances de l'autre (le personnel soignant), il ne peut qu'en résulter un conflit qui se caractérise par de la méfiance de part et d'autre. La méfiance du médecin s'exprime par un doute quant à l'existence réelle de la douleur et la méfiance de la personne souffrante se caractérise par le doute quant à la possibilité d'obtenir un soulagement adéquat.

Les processus subjectifs associés tant à l'expression de la douleur qu'à sa perception sont problématiques. Ils deviennent encore plus importants dans le cas de personnes présentant des atteintes cognitives, puisqu'elles ne sont même pas en mesure d'exprimer clairement leur douleur.

\section{LA DOULEUR DES PERSONNES AYANT DES ATTEINTES COGNITIVES}

La section précédente a fait ressortir que le soulagement de la douleur passe par une expression adéquate de la douleur ressentie et par la perception correcte de cette douleur par le personnel soignant. Les

préjugés ou les croyances de la part du personnel à l'égard de la douleur ou des gens qui l'expriment deviennent d'autant plus importants que la personne souffrante ayant des atteintes cognitives peut plus difficilement exprimer verbalement sa douleur. Wary, Pandolfo et Farnetti ${ }^{15}$ illustrent bien cette problématique. Ils soulignent que les personnes démentes meurent un jour, plus ou moins seules ou isolées, et que le bien-être et le confort que ressentent ces personnes ne sont pas toujours bien évalués, surtout en ce qui a trait à la douleur. Des croyances sont présentes à l'effet que les personnes atteintes de démence, tout particulièrement de la maladie d'Alzheimer, sont par ailleurs en bonne santé, commentaires que l'on entend souvent de la part du personnel soignant. Bien que la douleur ne soit pas un symptôme typique de la démence, il accompagne toutefois plusieurs maladies qui peuvent faire leur apparition chez les personnes ayant une démence. Comme le soulignent Boisvert et $\mathrm{Hall}^{16}$,

\section{LORSQUE LE SYSTÈME DE CROYANCES DE LA PERSONNE QUI SOUFFRE}

S'OPPOSE À CELUI DU PERSONNEL SOIGNANT, IL NE PEUT QU'EN RÉSULTER UN CONFLIT EXPRIMÉ PAR LA MÉFIANCE DE PART ET D'AUTRE.

chez les malades débilités, le moindre inconfort est perçu comme de la douleur indiquant ainsi un abaissement du seuil de la douleur. Les personnes souffrant d'une maladie en plus d'être atteintes de démence ne peuvent que présenter aussi cet abaissement du seuil de douleur. Cette situation est vraie aussi pour les personnes ayant un diagnostic de syndrome de Down, qui en plus des limitations cognitives déjà existantes, montrent un niveau de prévalence élevé de la démence de type Alzheimer ${ }^{17}$.

Pour l'ensemble des personnes présentant des déficits cognitifs (démence, déficience intellectuelle), les troubles de la communication sont fréquents et il peut donc être difficile pour elles d'exprimer leur douleur. Ross et Crook $^{18}$ mentionnent que les personnes âgées avec des problèmes d'attention, de mémoire et de raisonnement peuvent avoir de la difficulté à localiser la douleur ou même à la décrire. Ces auteures ajoutent que les comportements d'agressivité de cette clientèle résultent peut-être en partie de la difficulté qu'elle éprouve à bien identifier la source de son inconfort. Oliver et al. ${ }^{19}$ réalisent un parallèle intéressant pour supporter cette hypothèse en précisant que, dans plusieurs cas, les comportements d'automutilation et d'agression peuvent être diminués chez des personnes présentant des retards de développement cognitif grâce à l'apprentissage d'habiletés de communication et à l'amélioration de leur qualité de vie. Il est alors possible de penser que pour réduire les troubles de comportements associés à l'inconfort causé par une douleur physique, il faut les aider à bien l'exprimer.

Il faut donc trouver des moyens de pallier la difficulté qu'ont certaines personnes à exprimer clairement leur douleur. Pour la clientèle ayant des atteintes cognitives, une analyse multifactorielle impliquant différents partenaires en étroite collaboration est souvent nécessaire pour compenser ces difficultés ${ }^{20}$.

Dans la perspective de l'évaluation de la douleur des personnes atteintes de démence, Wary et al. ${ }^{21}$ font remarquer que les outils existants pour tenter de juger de l'intensité et de la nature de la douleur demandent une participation active de la personne qui souffre dans l'évaluation de sa douleur. Or, comme le souligne Fischer ${ }^{22}$, chez les personnes qui allient troubles de la conscience, troubles de la mémoire et difficultés de communication, il est impensable d'utiliser l'interrogation directe de la personne pour évaluer sa douleur.

Selon Janicki et Dalton ${ }^{23}$, une évaluation comparative de la capacité fonctionnelle et des comportements adaptatifs de la personne avec des déficiences cognitives peut informer sur l'environnement, sur l'évolution des comportements et sur les causes possibles du vécu douloureux. Pour la clientèle ayant une déficience intellectuelle, il existe des outils permettant de procéder à l'évaluation des comportements adaptatifs et des capacités fonctionnelles ainsi que des outils pour évaluer la qualité de vie. Ces outils peuvent donner quelques indications sur les éléments pouvant engendrer la douleur.

Plus directement en lien avec l'évaluation de la douleur, Wary et al. ${ }^{24}$ ont mis au point un outil qui est basé uniquement sur les manifestations observables de la douleur, le DOLOPLUS. Cette échelle évalue les signes somatiques (position antalgique au repos, mimique, protection des zones douloureuses, plaintes [cris ou 
gémissements] lors de l'examen clinique, etc.), les signes psychomoteurs (limitations dans les activités de la vie quotidienne) et les signes psychosociaux (troubles psychocomportementaux, problèmes de sommeil, etc.). Les auteurs tiennent à préciser que l'utilisation de cet outil vise simplement à répondre à la question: "Cette personne a-t-elle mal? » et ne permet pas d'emblée de déterminer la cause de la douleur. C'est ainsi que la détection de signes indiquant la présence d'une douleur peut amener le personnel soignant à choisir un traitement et à évaluer si le traitement fait diminuer les signes douloureux observés. L'objectif premier est alors de chercher à maintenir la personne dans un état de confort. Ces signes peuvent également servir de point de départ pour tenter de mettre en lumière la condition physique susceptible d'engendrer cette douleur et pour évaluer si elle est réversible ou non.

Une psychologue, travaillant auprès de la clientèle démente, insiste pour souligner que le DOLOPLUS est un des rares outils permettant de justifier la demande de prescriptions d'analgésiques pour les personnes ayant de graves problèmes cognitifs ${ }^{25}$. La limite ou les difficultés qu'elle souligne toutefois, lors de l'utilisation de l'instrument avec les personnes démentes, est le fait que les symptômes démentiels peuvent engendrer des réactions qui ressemblent à celles que susciterait la douleur, sans qu'il soit possible de distinguer l'une de l'autre. Par exemple, le refus de toute vie sociale, des troubles du comportement permanents, l'absence de communication, des troubles d'insomnie et une mimique inexpressive (regard vide), qui, selon le DOLOPLUS, constituent des signes probables de douleur, sont aussi des aspects présents chez les personnes ayant une démence, en l'absence de toute douleur physique. Toutefois, encore ici, même s'il est parfois difficile de savoir si les observations faites témoignent de la présence d'une douleur ou non, le simple fait de se poser la question permet de chercher une raison possible à ce qui semble être une douleur et, si c'est le cas, d'y remédier.

La diminution des capacités de communication et le traitement de la douleur constituent un défi de taille pour les systèmes de santé et le personnel soignant ${ }^{26}$. Il faut en premier lieu que le personnel soignant soit sensibilisé à la présence possible de la douleur. Si le personnel soignant doute de la présence réelle de la douleur, cela influencera leur perception et cela risque de perpétuer l'écart observé entre la quantité de douleur existante et la quantité d'action pour la soulager. Le double isolement s'installe alors pour la clientèle, isolement parce que la communication avec l'entourage est difficile et isolement dans la douleur, sans que la personne puisse comprendre quoi faire pour s'en libérer. Pour le personnel soignant, la moitié du chemin est déjà parcourue dès que la question suivante est posée: "Cette personne a-t-elle mal ? » Si en parallèle se développent des outils pour évaluer adéquatement cette douleur, il y a un peu d'espoir de briser l'isolement dans lequel se trouve la personne souffrante ayant des déficits cognitifs. Ainsi, à la «mort de l'esprit» que peut engendrer la présence des atteintes cognitives chez ces personnes s'ajoute le non-soulagement de la douleur physique, ce qui a pour effet d'emmurer encore plus la personne dans un monde à part.

\section{Notes}

1 C.R. CHAPMAN et J. GAVRIN, "La souffrance et sa relation avec la douleur ", dans Douleur et antalgie, Les annales de soins palliatifs, vol. 2, sous la direction de D.J. ROY et C.-H. RAPIN, Montréal, Collection Amaryllis, 1993, p. 3-22.

2 P. ROSSMAN, Hospice, New York, Fawcette Columbine, 1977, 238 pages.

3 D. ROY, "Le contrôle de la douleur: un problème éthique ou une obligation morale ", Les annales de soins palliatifs, vol. 2, sous la direction de D. J. ROY et C.-H. RAPIN, Montréal, Collection Amaryllis, 1993, p. 33-42.

4 A. BARREAU, Humaniser la mort, Paris, Éditions l'Harmattan, 1993, 186 pages.

5 J.R. DAVITZ et L.L. DAVITZ, Inferences of patient's pain and psychological distress, New York, Springer, 1981, 209 pages.

6 S. BOUILLÉ, «La douleur de l'âge ou l'âge de la douleur», dans Quand vieillir fait mal, Les cahiers des Journées de formation annuelle du Sanatorium Bégin, vol. 12, sous la direction du Sanatorium Bégin, LacEtchemin (Québec), 1993, p. 43-55.

7 R. MELZACK et P.D. WALL, Le défi de la douleur, Paris, Vigot, 1989, 240 pages.

8 J.R. DAVITZ et L.L. DAVITZ, ibid. note 5.

9 J.-Y. VISIER, «L'enfant douloureux», dans La dimension de la souffrance chez le malade douloureux chronique, sous la direction de E. Ferragut, Paris, Masson, 1995, 91-102.

10 S. BOUILLÉ, ibid. note 6.

11 A. COOK et R. ROY, "Attitudes, Beliefs, and Illness Behavior», dans Chronic Pain in Old Age, sous la direction de R. ROY, Toronto, University of Toronto Press, 1995, p. 20-37.

$12 \mathrm{~S}$. MURRAY et al. "Perception et soulagement de la douleur: Perception de la douleur et intention de la soulager chez de futurs médecins », Interactions, vol. 2, no 2, 1998, p. 269-284.

13 M. M. ROSS et J.M. CROOK, «Pain in Later Life: Present State of Knowledge », dans Chronic Pain in Old Age, sous la direction de R. ROY, Toronto, University of Toronto Press, 1995, p. 3-19.
14 A. COOK et R. ROY, ibid. note 11.

15 B. WARY et al., "Ce vieillard a-t-il mal ?», Les annales de soins palliatifs, vol. 2, sous la direction de D.J. ROY et C.-H. RAPIN, Montréal, Collection Amaryllis, 1993, p. 89-98.

16 M. BOISVERT et P. HALL, "Soins palliatifs ", dans Précis de gériatrie, sous la direction de M. ARCAND, R. HÉBERT, St-Hyacinthe, Edisem, 1998, p. 865-880.

17 C. OLIVER et al., "Cognitive Deterioration in Adults with Down Syndrome: Effects on the Individual, Caregivers, and Service Users ", American Journal on Mental Retardation, vol. 105, no 6, 2000, p. 455-464.

18 M. M. ROSS et J.M. CROOK, ibid. note 13.

19 C. OLIVER et al., ibid. note 17.

20 F. DANTO, La douleur chez la personne âgée en difficulté de communication: Retentissement sur l'équipe soignante, U.F.R. Santé, médecine et biologie humaine, Paris, 1999 (http://www.multimania.com/danto/ memoire/memoirehtml.htm).

21 B. WARY et al., ibid. note 15.

22 M.-C. FISCHER, «Évaluer la douleur chez la personne démente », Alzheimer, vol. 1, no 2, décembre 1998, p. 19.

23 M.-P. JANICKI et A.J. DALTON, «Prevalence of Dementia and Impact on Intellectual Disability Service», Mental Retardation, vol. 38, no 3, 2000, p. 276-288.

24 B. WARY et al., ibid. note 15 . Voir aussi le site: www.doloplus.com

25 M.-C. FISCHER, ibid. note 22.

26 M.-P. JANICKI et A.J. DALTON, ibid. note 23. 